\title{
Sequelas da Covid-19: uma reflexão sobre os impactos na saúde do trabalhador
}

\author{
Sequelae of Covid-19: a reflection on the impacts on the health of the worker \\ Secuelas de Covid-19: una reflexión sobre los impactos en la salud de los trabajadores
}

Recebido: 16/10/2021 | Revisado: 20/10/2021 | Aceito: 21/10/2021 | Publicado: 24/10/2021

\author{
Bianca Fontana Aguiar \\ ORCID: https://orcid.org/0000-0001-9054-8245 \\ Universidade Federal do Paraná, Brasil \\ E-mail: biancafoguiar@hotmail.com \\ Leila Maria Mansano Sarquis \\ ORCID: https://orcid.org/0000-0002-0542-5062 \\ Universidade Federal do Paraná, Brasil \\ E-mail: 1mmsarquis@gmail.com \\ Fernanda Moura D'Almeida Miranda \\ ORCID: https://orcid.org/0000-0001-7140-9557 \\ Universidade Federal do Paraná, Brasil \\ E-mail: fmdmiranda@gmail.com
}

\begin{abstract}
Resumo
Objetivo: realizar uma reflexão sobre como as sequelas da Covid-19 podem impactar na saúde do trabalhador. Método: Trata-se de uma reflexão. Os artigos selecionados foram organizados por meio dos eixos temáticos: Sequelas da Covid-19 e Impactos na saúde do trabalhador. Resultados: A Covid-19 é uma doença com aspectos ainda a serem revelados, um deles são as sequelas na saúde da população. Esta condição é preocupante, visto o risco de uma sobrecarga nos sistemas de saúde. As complicações da Covid-19 são inúmeras e capazes de interferirem na saúde do trabalhador, seja por meio da dificuldade no exercício ocupacional ou até por ocasionarem aposentadorias precoces. Acredita-se que algumas sequelas são tratáveis, entretanto podem perdurar por algum tempo; em outros casos, pode haver sequelas irreversíveis. Conclusão: Faz-se necessário estabelecer políticas públicas de saúde, previdenciária e trabalhista que garantam aos trabalhadores tratamento de saúde adequado, estabilidade no emprego ao retorno benefício social.
\end{abstract}

Palavras-chave: Infecções por Coronavírus; Covid-19; Saúde do trabalhador; Política de saúde do trabalhador.

\begin{abstract}
Objective: to reflect on how the sequelae of Covid-19 can impact workers' health. Methods: This is a reflection. The selected articles were organized according to the thematic axes: Sequelae of Covid-19 and Impacts on workers' health. Results: Covid-19 is a disease with aspects yet to be revealed, one of which is the consequences on the health of the population. This condition is worrisome, given the risk of overloading health systems. The complications of Covid-19 are capable and capable of interfering with the worker's health, whether through difficulties in occupational exercise or even because they cause early retirement. It is believed that some sequelae are treatable, however, they can be lost for some time, in other cases, there may be irreversible sequelae. Conclusion: It is necessary to establish public health, social security and labor policies that guarantee workers adequate health treatment, job stability and social benefits.
\end{abstract}

Keywords: Coronavirus infections; Covid-19; Occupational health; Occupational health policy.

\section{Resumen}

Objetivo: reflexionar sobre cómo las secuelas de Covid-19 pueden afectar la salud de los trabajadores. Métodos: esta es una reflexión. Los artículos seleccionados se organizaron según los ejes temáticos: Secuelas de Covid-19 e Impactos en la salud de los trabajadores. Resultados: Covid-19 es una enfermedad con aspectos aún por revelar, uno de los cuales son las consecuencias en la salud de la población. Esta condición es preocupante, dado el riesgo de sobrecargar los sistemas de salud. Las complicaciones del Covid-19 son capaces y capaces de interferir en la salud del trabajador, ya sea por dificultades en el ejercicio ocupacional o incluso porque provocan la jubilación anticipada. Se cree que algunas secuelas son tratables, sin embargo, pueden perderse por algún tiempo, en otros casos, puede haber secuelas irreversibles. Conclusión: Es necesario establecer políticas de salud pública, seguridad social y laboral que garanticen a los trabajadores un adecuado tratamiento de salud, estabilidad laboral y beneficios sociales.

Palabras clave: Infecciones por Coronavirus; COVID-19; Salud del trabajador; Política de salud ocupacional. 


\section{Introdução}

No final de 2019, foi registrado na cidade de Wuhan, na China, um surto de pneumonia de etiologia até então desconhecida. Tratava-se de uma nova cepa de coronavírus, o qual foi denominado de Síndrome Respiratória Aguda Grave do Coronavírus 2 (SARS-Cov-2) (Yang et al., 2020). Com elevada taxa de infectividade, o novo coronavírus se disseminou rapidamente por diversos países atingindo grande número da população mundial e ocasionando a Coronavirus disease - 2019 (Covid-19) (Gonçalves et al., 2020).

Devido a emergência de Saúde Pública, em março de 2020, a Organização Mundial da Saúde (OMS), declarou a situação como uma pandemia (World Health Organization, 2020a). No mundo, o número de casos confirmados da Covid-19, até final do mês de setembro de 2021, eram mais de 200 milhões e quatro milhões de óbitos; apenas no Brasil os dados registraram mais de 21 milhões de casos confirmados e 588.597 mil óbitos (Paraná, 2021).

A Covid-19 caracteriza-se como uma doença, cujos sintomas podem se manifestar em graus leves, moderados e graves, sendo considerada uma doença sistêmica que atinge vários órgãos e pode gerar complicações que podem resultar em óbitos (Nice, 2020). Em média, os sintomas da infecção aguda se normalizam em até 14 dias, no entanto alguns deles podem ser persistentes e permanecem em determinados pacientes, com probabilidade do surgimento de algumas e diferentes sequelas a longo prazo (Nice, 2020).

A Covid-19 longa representa uma série de sintomas e complicações prolongadas, que vão além do período inicial da doença ou que aparecem no período de pós infecção (Willi et al., 2021). Embora haja indícios dos efeitos a longo prazo da Covid-19, ainda são preocupantes a maneira como essas condições podem afetar a população infectada.

Estas sequelas têm potencial de comprometer as atividades laborais e prejudicar a saúde do trabalhador, em virtude de a grande maioria da população, que está ou esteve em contato com o vírus da Covid-19, ainda permanece ativa no mercado de trabalho (Xiong et al., 2021). Assim, torna-se necessário um olhar para esta questão, principalmente para o desenvolvimento de ações de acompanhamento e cuidado com a saúde dos trabalhadores. Deste modo, o objetivo deste estudo é realizar uma reflexão a respeito de como as sequelas da Covid-19 podem impactar na saúde do trabalhador.

\section{Metodologia}

Trata-se de um estudo reflexivo, realizado entre junho e setembro de 2021, cuja questão norteadora foi analisar como as sequelas da Covid-19 podem impactar na saúde do trabalhador. Para subsidiar a construção desta reflexão, utilizou-se de evidências científicas nacionais e internacionais, com enfoque na temática escolhida.

Para isto foi realizada uma busca nas bases de dados eletrônicas Literatura Latino Americana e do Caribe em Ciências e Saúde (LILACS), Medical Literature Analysis and Retrieval System Online (MEDLINE/PubMed) e no Portal Regional da Biblioteca Virtual em Saúde (BVS). Os descritores em Ciências da Saúde (DeCS) utilizados foram: "Covid-19”, "SARS-COV2", “Complications”, “Occupational health”. Teve-se como critérios de inclusão: artigos disponíveis na íntegra, em português, inglês e espanhol, publicados no período de 2020 a agosto de 2021 e que respondessem ao objetivo do estudo.

Assim, identificou-se 152 publicações, em que 16 foram elegíveis para este manuscrito e foram organizadas por meio de dois eixos temáticos: Sequelas da Covid-19 e Impactos na saúde do trabalhador.

\section{Resultados e Discussão}

\section{Sequelas da Covid-19}

A Covid-19 é uma doença com muitos aspectos ainda a serem revelados, um deles são as sequelas na saúde da população que se infectou pelo SARS-Cov-2 que podem surgir a longo prazo (Korompoki et al., 2021). Denominou-se tal 
situação de Covid-19 longa, em virtude de os efeitos de alguns sintomas permanecerem por mais de 12 semanas desde o início da infecção, mas ainda não se sabe por quanto tempo eles podem persistir e o nível de complexidade que esses podem gerar (Korompoki et al., 2021). No entanto, esta condição de efeitos a longo prazo é preocupante, visto o potencial risco de uma sobrecarga nos sistemas de saúde em busca de atendimento para o tratamento das complicações decorrentes da Covid-19.

Ademais, a realidade brasileira tem demonstrado que a maioria dos acometidos entre os adultos em idade produtiva são enfermeiros, técnicos de enfermagem, médicos, demais profissionais de saúde, motoristas, cobradores de ônibus, recepcionistas, auxiliares administrativos, caixas de supermercados, entre outros, que são considerados trabalhadores de serviços essenciais e necessitam deslocar-se para o sustento de sua família e para manter o funcionamento de sua ocupação.

Neste contexto, ressalta-se que as sequelas da Covid-19 podem ocasionar impactos sociais e econômicos a sociedade brasileira, visto que esses trabalhadores necessitam de afastamento do trabalho para tratamento de saúde e acabam gerando uma sobrecarga aos colegas de trabalho e/ou um déficit de trabalhadores para essas áreas. Ainda, muitas vezes esses trabalhadores não têm o conhecimento da relação da Covid-19 com o trabalho, o que pode acarretar dificuldades ao trabalhador para solicitar benefícios previdenciários e trabalhistas para o tratamento de saúde.

Entre as possíveis sequelas, apontam-se as neurológicas, respiratórias, psicológicas, vasculares, gastrointestinais, cutâneas, e até as relacionadas ao olfato e paladar. Identificou-se em um estudo que os principais sintomas apresentados no pós-Covid foram cefaleia, tontura, náusea, confusão mental, mialgia, distúrbios vasculares e algumas consequências graves, como hemorragia intracerebral aguda, trombose de seio venoso cerebral, encefalopatia e síndrome de Guillain-Barré (Colantes et al., 2021). Percebe-se que as complicações neurológicas são as que mais acometeram a população que teve Covid-19, o que exige dos profissionais de saúde um olhar atento para tais agravos, em busca de uma intervenção efetiva e precoce.

Observou-se em uma revisão sistemática que $80 \%$ dos indivíduos que tiveram diagnóstico positivo para Covid-19 continuaram com pelo menos um sintoma após a fase aguda da doença, sendo a fadiga, o mais prevalente, principalmente entre as mulheres. Outros sintomas foram: tosse persistente, dispneia pós-esforço, distúrbios relacionados ao sono, cefaleia, anosmia e vertigem (Lopez-Leon et al., 2021). Como as sequelas da doença ainda estão sendo pesquisadas, há a possibilidade da existência de outras manifestações clínicas pós-covid, tais como: alopecia, perda de olfato ou paladar, entre outras, o que reforça a necessidade de novos estudos sobre essa temática.

Em outro estudo realizado na Itália, com 143 pacientes no período pós-covid, revelou que 87,4 \% deles, persistiam com um ou mais sintomas, sendo relatado a presença de fadiga (53,1\%), dispneia (43,4\%), dores nas articulações $(27,3 \%)$ e dores no peito (21,7\%) (Carfì, Bernabei \& Landi, 2020). Resultado que corrobora com estudo realizado nos Estados Unidos, o qual refere que entre 177 pessoas, foram relatados sintomas persistentes da Covid-19 por 26,6\% com idades de 18 a 39 anos, em 30,1\% entre 40 e 64 anos e $43,3 \%$ com mais de 65 anos, entre eles estavam a fadiga, perda de olfato ou paladar, perda de memória e dificuldade de concentração (Logue et al., 2021).

Destaca-se entre as sintomatologias pós a Covid-19, a chamada "névoa do cérebro", a qual consiste na presença de fadiga, falta de concentração e até de dificuldade de memorização (Stefano, 2021). Salienta-se que o aparecimento desses eventos interfere diretamente no desempenho profissional, muitas vezes desenvolvendo incapacidades permanentes. Essas sequelas podem trazer comprometimento aos trabalhadores que precisam realizar tratamentos de saúde e por muitas vezes necessitam afastar-se do trabalho. Esse afastamento traz um aumento de demanda aos serviços previdenciários e acarreta uma sobrecarga financeira, tanto para os trabalhadores como para a sociedade em geral.

Além disso, os serviços de saúde já esgotados necessitam organizar-se para o atendimento desses trabalhadores portadores de sequelas de Covid-19 e por muitas vezes esses serviços não dispõe de recursos humanos e financeiros para esse tipo de atendimento, uma vez que estas sequelas ainda estão em estudos e seu tratamento é obscuro e ainda novo. Faz-se 
necessário uma estruturação dos serviços de saúde e previdenciários para atendimento desses trabalhadores para que esses possam retornar a sua vida familiar e laboral em condições de saúde adequadas.

Outro fato preocupante é a probabilidade do surgimento de problemas de saúde mental, como ansiedade e depressão, não só decorrentes da pandemia, como pelo isolamento social, mas oriundos da própria infecção (Szcześniak, Gładka, Misiak, Cyran, \& Rymaszewska, 2021). É notável que as questões relacionadas a saúde mental se tornaram mais evidentes neste cenário, o que podem acarretar prejuízos para população e a sociedade.

A pandemia tem causado a elevação no número de desempregados, o aumento da pobreza e das desigualdades sociais, tornando necessário investimentos em políticas econômicas e de saúde pública que possam contribuir para minimizar os efeitos devastadores da pandemia na sociedade brasileira. Ainda, em relação à saúde mental é importante garantir o acesso da população aos serviços de saúde de qualidade e gratuitos, a fim de promover um aporte emocional e social.

\section{Impactos para a saúde do trabalhador}

A Covid-19 é caracterizada como uma doença relacionada ao trabalho, por considerar que diversos trabalhadores estão expostos, no seu local de ocupação, ao risco de contaminação pelo SARS-CoV-2 (Bahia, 2020). Cabe destacar que para estabelecer a relação da Covid-19 com o trabalho, deve-se investigar os históricos da doença e da situação ocupacional do trabalhador (Bahia, 2020). O reconhecimento dessa situação é fundamental, pois o fato de os trabalhadores saírem de suas residências para cumprir com suas atividades laborais, já os coloca em risco (Maeno, 2021). Por hora, há apenas a classificação da Covid-19, segundo a Classificação Estatística Internacional de Doenças e Problemas Relacionados com a Saúde (CID-10), a qual pertence ao grupo U07.1, quando houve confirmação do vírus e U07.2 quando a doença foi diagnosticada clinicamente e epidemiologicamente (World Health Organization, 2020b).

No Brasil, apenas entre os trabalhadores da saúde, 112.997 tiveram casos confirmados para Covid-19, de acordo com a Classificação Brasileira de Ocupações (CBO); as profissões com maior número de adoecidos foram auxiliares e técnicos de enfermagem com mais de 33.338 casos, seguido de enfermeiros com 19.053 e médicos com 12.002 (Brasil, 2021). Destaca-se que a equipe de enfermagem é a área com maior número de funcionários na área de saúde e responsável pelo cuidado integral do paciente, consequentemente os que estão mais expostos a adoecerem pela Covid-19 (Gómez-Ochoa et al., 2020).

No que concerne para a saúde do trabalhador, os impactos das sequelas da Covid-19 são diversos, um exemplo é relatado em um estudo realizado na Itália, em que 60 dias após o início dos sintomas da Covid-19, entre 143 pacientes, somente $12,6 \%$ referiam estar sem nenhum sintoma da doença, o restante ainda apresentava sintomas, com relatos de piora da Qualidade de Vida (QV) entre 44,1\% dos pacientes (Carfì, Bernabei \& Landi, 2020). Diante disso, observa-se a diminuição da QV entre a população convalescente, o que modifica o dia a dia, interfere nas atividades diárias e capacidade de trabalho.

Evidenciou-se em outro estudo, que dentre 177 participantes, os sintomas persistentes da Covid-19 foram relatados por 26,6\% dos pacientes com idades entre 18 e 39 anos, 30,1\% entre 40 a 64 anos e 43,3\% com 65 anos ou mais, e os acometidos por essa situação relataram apresentar dificuldade em pelo menos uma atividade de vida diária (AVD) e baixa produtividade (Logue et al., 2021). Os dados apontam que a maior parte da população ativa no mercado de trabalho, com faixaetária de 18 a 64 anos, estão suscetíveis a terem alguma manifestação no pós-Covid e isto pode afetar no desempenho ocupacional. Destaca-se que nas empresas que possuem Serviços Especializados em Segurança e Medicina do Trabalho faz-se necessário implementar ambulatórios de acompanhamentos aos trabalhadores acometidos pela Covid-19 para observar as sequelas relatadas e realizar os encaminhamentos necessários para tratamentos de saúde e assistência previdenciária.

Uma pesquisa realizada nos Estados Unidos, acompanhou uma coorte de 177 indivíduos por até nove meses, após os primeiros sintomas da doença, aproximadamente $30 \%$ deles relataram sintomas persistentes, não terem retornado ao seu estado normal de saúde e dificuldade de retornar ao trabalho (Tenforde et al., 2020). Neste contexto, é fundamental que os serviços de 
saúde planejem e realizem o acompanhamento a longo prazo de pacientes que tiveram Covid-19 (Huang et al., 2020), outrossim priorizem ações voltadas para recuperação da saúde do trabalhador.

Dessa forma, os profissionais de saúde precisam estar preparados para o atendimento desta população e devem atentar-se para o reconhecimento de sintomas que correspondam a sequelas da Covid-19, bem como observar e registrar o tempo de permanência deles (Wang, Kream, \& Stefano, 2020). Como a pandemia continua em ascensão, o número de pacientes que venham a apresentar sequelas em saúde só tende a aumentar. As empresas contratantes e as instituições públicas devem repensar a assistência à saúde do trabalhador a fim de garantir qualidade no atendimento de saúde prestado para que o trabalhador possa retornar a suas atividades cotidianas e laborais em pleno estado de saúde.

Ressalta-se a necessidade do conhecimento de como estes agravos podem influenciar nas condições de saúde e nas questões relacionadas ao trabalho (Campos et al., 2020). No Brasil, faz-se necessário instituir políticas públicas de saúde e assistenciais que garantam a manutenção da saúde e do emprego dos trabalhadores sequelados pela Covid-19 e que apresentem alguma restrição as atividades de trabalho desempenhadas anteriormente. Acredita-se que algumas sequelas são tratáveis, entretanto podem perdurar necessitando das empresas contratantes um retorno gradual as funções desempenhadas. Em outros casos, pode haver sequelas irreversíveis em que os trabalhadores necessitem de uma mudança de função ou readaptação funcional, nesses casos as empresas contratantes devem garantir as adequações para que os trabalhadores possam retornar as atividades laborais e assim possam continuar mantendo seu sustento.

Pode-se observar que as complicações da Covid-19 são inúmeras e capazes de interferirem na saúde do trabalhador, seja por meio da dificuldade no exercício da função ocupacional, ou até por ocasionarem aposentadorias precoces. Este cenário é desafiador e requer resposta imediata de políticas públicas e serviços de saúde. Ainda, é necessário destacar que a Covid-19, em muitos casos está relacionada ao trabalho e seu reconhecimento como uma doença ocupacional garante ao trabalhador benefícios previdenciários e trabalhistas que podem auxiliar no tratamento das sequelas ocasionadas pela Covid-19.

O estudo apresentou como limitação o fato de ainda não haver publicações que possam predizer, com certeza, a duração da Covid-19 longa e de todos as complicações que ela pode ocasionar aos trabalhadores. No entanto, essa reflexão contribui com o desenvolvimento prioritário de iniciativas que visem o restabelecimento da saúde dos inúmeros trabalhadores acometidos pela Covid-19. Assim como, subsidia o desenvolvimento de pesquisas futuras e reflexões relacionadas a saúde do trabalhador.

\section{Conclusão}

Ao refletir sobre como as sequelas da Covid-19 podem impactar na saúde do trabalhador, observou-se que as manifestações a longo prazo são diversas e que os sintomas variam desde os neurológicos, respiratórios e alterações na saúde mental. Essas sequelas podem estar relacionadas ao trabalho, visto que a maioria da população acometida pela Covid-19 encontra-se em idade produtiva.

As sequelas da Covid-19 interferem na piora da Qualidade de Vida dificultando as atividades diárias e diminuindo a produtividade. As empresas contratantes e as instituições públicas devem garantir aos trabalhadores um retorno gradual a suas atividades laborais, a fim de diminuir o impacto desse agravo a saúde destes.

Faz-se necessário estabelecer políticas públicas de saúde, previdenciária e trabalhista que garantam aos trabalhadores acometidos por sequelas da Covid-19 tratamento de saúde adequado, estabilidade no emprego ao retorno e benefício social em caso de necessidade de afastamento do trabalho. Algumas instituições de saúde, já estão organizadas para o atendimento desta população, com serviços de reabilitação e acompanhamento; no entanto, espera-se que os serviços de saúde e as instituições empregadoras reconheçam a Covid-19 relacionada ao trabalho para que os trabalhadores possam ter seus direitos trabalhistas e previdenciários garantidos. 


\section{Referências}

Bahia. (2020). Secretaria da Saúde do Estado. Superintendência de Vigilância e Proteção da Saúde. Diretoria de Vigilância e Atenção à Saúde do Trabalhador. Centro Estadual de Referência em Saúde do Trabalhador. Orientações técnicas para a investigação e notificação de casos de Covid-19 relacionados ao trabalho. Salvador, BA.

Brasil. (2021). Ministério da Saúde. Secretaria de Vigilância em Saúde. Boletim Epidemiológico Especial. Brasília, DF. https://www.gov.br/saude/ptbr/media/pdf/2021/junho/18/boletim_epidemiologico_covid_67.pdf

Campos, M. R., Schramm, J. M. D. A., Emmerick, I. C. M., Rodrigues, J. M., Avelar, F. G. D., \& Pimentel, T. G. (2020). Carga de doença da COVID-19 e de suas complicações agudas e crônicas: reflexões sobre a mensuração (DALY) e perspectivas no Sistema Único de Saúde. Cadernos de Saúde Pública, 36, e00148920.

Carfi, A., Bernabei, R., \& Landi, F. (2020). Persistent symptoms in patients after acute COVID-19. Jama, 324(6), 603-605.

Collantes, M. E. V., Espiritu, A. I., Sy, M. C. C., Anlacan, V. M. M., \& Jamora, R. D. G. (2021). Neurological manifestations in COVID-19 infection: a systematic review and meta-analysis. Canadian Journal of Neurological Sciences, 48(1), 66-76.

Gómez-Ochoa, S. A., Franco, O. H., Rojas, L. Z., Raguindin, P. F., Roa-Díaz, Z. M., Wyssmann, B. M., ... \& Muka, T. (2021). COVID-19 in health-care workers: a living systematic review and meta-analysis of prevalence, risk factors, clinical characteristics, and outcomes. American journal of epidemiology, 190(1), 161-175.

Gonçalves, C. W. B., Gomes, D. L. F., Neto, A. B. P., Lima, G. S., Reis, K. H. J. F., \& Cláudio, E. S. (2020). Incidence of COVID-19 in the states of the Northern Region of Brazil. Revista Prevenção de Infecção e Saúde, 6.

Huang, C., Huang, L., Wang, Y., Li, X., Ren, L., Gu, X., ... \& Cao, B. (2021). 6-month consequences of COVID-19 in patients discharged from hospital: a cohort study. The Lancet., 397: 220-32.

Korompoki, E., Gavriatopoulou, M., Hicklen, R. S., Ntanasis-Stathopoulos, I., Kastritis, E., Fotiou, D., ... \& Kontoyiannis, D. P. (2021). Epidemiology and organ specific sequelae of post-acute COVID19: A Narrative Review. Journal of Infection.

Logue, J. K., Franko, N. M., McCulloch, D. J., McDonald, D., Magedson, A., Wolf, C. R., \& Chu, H. Y. (2021). Sequelae in adults at 6 months after COVID19 infection. JAMA network open, 4(2), e210830-e210830.

Lopez-Leon, S., Wegman-Ostrosky, T., Perelman, C., Sepulveda, R., Rebolledo, P. A., Cuapio, A., \& Villapol, S. (2021). More than 50 Long-term effects of COVID-19: a systematic review and meta-analysis.

Maeno M. (2021). Doenças ocupacionais relacionadas à pandemia de Covid-19: fatores de risco e prevenção. https://basis.trt2.jus.br/bitstream/handle/123456789/13988/maeno_maria_doencas_ocupacionais.pdf?sequence=4\&isAllowed=y

Nice. (2020). COVID-19 rapid guideline: managing the long-term effects of COVID-19. https://www.nice.org.uk/guidance/ng188

Paraná. (2021). Secretaria de Estado da Saúde do Paraná. Informe Epidemiológico. https://www.saude.pr.gov.br/Pagina/Coronavirus-COVID-19

Stefano, G. B. (2021). Historical insight into infections and disorders associated with neurological and psychiatric sequelae similar to long COVID. Medical Science Monitor: International Medical Journal of Experimental and Clinical Research, 27, e931447-1.

Szcześniak, D., Gładka, A., Misiak, B., Cyran, A., \& Rymaszewska, J. (2020). The SARS-CoV-2 and mental health: From biological mechanisms to social consequences. Progress in Neuro-Psychopharmacology and Biological Psychiatry, 110046.

Tenforde, M. W., Kim, S. S., Lindsell, C. J., Rose, E. B., Shapiro, N. I., Files, D. C., ... \& IVY Network Investigators. (2020). Symptom duration and risk factors for delayed return to usual health among outpatients with COVID-19 in a multistate health care systems network-United States, March-June 2020. Morbidity and Mortality Weekly Report, 69(30), 993.

Wang, F., Kream, R. M., \& Stefano, G. B. (2020). Long-term respiratory and neurological sequelae of COVID-19. Medical science monitor: international medical journal of experimental and clinical research, 26, e928996-1.

Willi, S., Lüthold, R., Hunt, A., Hänggi, N. V., Sejdiu, D., Scaff, C., \& Schlagenhauf, P. (2021). COVID-19 sequelae in adults aged less than 50 years: a systematic review. Travel medicine and infectious disease, 101995.

World Health Organization (2020). Emergencies. Diseases. Coronavirus disease (COVID-19) Pandemic. https://www.who.int/emergencies/diseases/novelcoronavirus-2019

World Health Organization (2020). Emergencies. Diseases. Diretrizes Internacionais para a certificação e Classificação (Codificação) da Covid-19 como causa de morte. https://www.who.int/classifications/icd/Guidelines_Cause_of_Death_COVID-19-20200420-PT_Apr_24.pdf?ua=1

Xiong, Q., Xu, M., Li, J., Liu, Y., Zhang, J., Xu, Y., \& Dong, W. (2021). Clinical sequelae of COVID-19 survivors in Wuhan, China: a single-centre longitudinal study. Clinical Microbiology and Infection, 27(1), 89-95.

Yang, W., Sirajuddin, A., Zhang, X., Liu, G., Teng, Z., Zhao, S., \& Lu, M. (2020). The role of imaging in 2019 novel coronavirus pneumonia (COVID-

19). European radiology, 1-9. 